\title{
Normalization in LCA: How to ensure consistency?
}

\author{
Arnaud Hélias ${ }^{1,2 *}$, Rémi Servien ${ }^{3,4}$ \\ ${ }^{1}$ ITAP, Univ Montpellier, INRAE, Institut Agro, Montpellier, France \\ ${ }^{2}$ Elsa, Research group for Environmental Lifecycle and Sustainability Assessment, Montpellier, France \\ ${ }^{3}$ INRAE, Univ. Montpellier, LBE, Narbonne, France \\ ${ }^{4}$ ChemHouse Research Group, Montpellier, France \\ Corresponding author : arnaud.helias@inrae.fr \\ (1)
}


decision-making process is an expectation. In the field of LCA, this justifies the normalization and weighting steps. The weight represents the importance of the criterion and its design is a real research topic in itself, but we are interested here in normalization.

\section{Method}

\subsection{Meaning of the normalization}

The normalization in the LCA framework is the division of the impact computed for the system under study, by the impact of a reference value named the normalization reference. LCA normalization has been identified as a leading driver in the aggregation process, with high consequences on the results (Myllyviita et al. 2014; Prado et al. 2019; Muhl et al. 2021).

Normalization has three main purposes (Pizzol et al. 2017): (1) to compare the results in order to check plausibility, (2) to facilitate communication, and (3) to be free of unit constraints for weighting, with the impacts expressed on a common scale. This last point focuses our attention here.

In LCA, normalization is also twofold (Laurent and Hauschild 2015): (1) internal if the normalization reference is defined on the basis of case studies, as the result of a system " $\mathrm{A}$ " expressed in proportion of the result of a system "B", and (2) external if an independent reference is used, as the results of systems " $\mathrm{A}$ " and "B" expressed in proportion to the average citizen year impact.

An internal normalization ensures, by definition, consistency between the system under study and the reference. However, it remains context-dependent, which is contrary to the objectives of LCA, and cannot be used with generic weighting (Norris 2001). An external normalization makes possible an aggregation process that is reproducible and independent of the study. However, two modelling processes are used, one for the system and the other for the reference. This raises questions about the representativeness and the consistency between both representations (Heijungs et al. 2007).

The normalization task (sometimes named standardization in some scientific communities) is not unique to LCA and is encountered by anyone who deals in data processing. For decision, not all, but most MADM models require a normalization step. The choice of the normalization method is often part of the MADM process and this affects the result: A different method of normalization will lead to a different result. There is no consensus on this step in the MADM community and Jahan and Edwards (2015) reviewed 31 methods (sum, norm, max-min, Z-transformation...). All of them are based on the systems under study and from LCA viewpoint, they are internal normalizations. LCA was initially defined outside the framework of the MADM (Pizzol et al. 2017) and the choice was made to mainly use external normalization: As a result, the 
aggregation process is not determined by the specifics of the study, contrary to most MADM methods. This is undoubtfully an advantage for the genericity, repeatability, and independence of the process.

Global external normalizations are now recommended in the guidelines (Verones et al. 2017). This is the most relevant system of reference: In our globalized economy, any functional unit involves processes across all continents and the problem has to be taken as a whole, using a normalization reference encompassing the entire world.

\subsection{Consistency issue}

LCA is a modelling work: For a functional unit, we draw up the associated impacts. This process is built with rules, and one of the most important is additivity: the impact of two functional units is the sum of the impacts of each. In this sense, external normalization means dividing the impact of the product or service (corresponding to the functional unit of the study) by the sum of all the products and services on a global scale (the reference). To ensure consistency in the modelling work, the way in which the products and services are represented should be the same. As an illustration, Figure 1 offers a simplistic schematic view, with the system under study being an apple and the reference for normalization being the world. From real systems, the modelling processes allow having representations, here blurred or partial. For the normalization, having a blur/blur or partial/partial ratio is preferable to a blur/partial or partial/blur one, whatever the soundness of the modelling.

The raw data and their processing must be identical both for the system under study and the normalization references (unless it can be proved that there is no bias or that the bias is identical). In other words, we can say that an LCA is a model of a part of the world and the reference is a model of the world. Models and choices must be consistent.

\subsubsection{Economic Input-Output LCA}

An EIO-LCA uses aggregated sectoral data and their interdependencies. Fractions of the impacts associated to the sectors of the economy are then attributed to the system under study with the Leontief's wellknown equation. With this top-down approach, we have the same model between the world (the totality of all economic sectors) and the system under study (parts of economic sectors). We can ensure consistency in the normalization process (subject to the calculating of this value from the EIO database). Unfortunately, the process-based LCA differs. 
A process-based LCA is more like a Lego ${ }^{\circ}$ construction. The LCA practitioners take "bricks" from a Life Cycle Inventory (LCI) database and assemble them to build the system under study. Sometimes, they create a "brick", but this is far from being their primary activity. For each type of "brick" (a component or a process, called a dataset by LCI database providers) that is required, the practitioners take a certain number of them (i.e. the final demand). With this bottom-up approach, we do not have the information to shape the world as a whole. All the datasets into the LCI database describe what constitutes the world, but the quantity of each is missing. As an example, we can consider that the dataset "market for apple | apple (GLO)" from ecoinvent (Wernet et al. 2016) allows us to have the average impact of an apple at the world level, but it does not allow us to know how many apples are produced in all the orchards of the world.

With only a process-based LCA database, we cannot calculate the LCA of human activity at the global level. The consistency of the normalization cannot be argued.

\section{$3 \quad$ Results}

\subsection{Normal versus log-normal laws}

In LCA, we normalize by the whole impact (or by a proportion of it when reduced to a citizen, but the reasoning remains the same). This is undeniably a relevant solution, the result being a fraction (studied system divided by the whole) that is easy to interpret and communicate. Divided by the sum of elements is one of the normalization identified by Jahan and Edwards (2015) but there are many others.

In data processing, looking at the law of data distribution to select the descriptors is always relevant. The best known is the normal distribution law, described by the arithmetic mean and the standard deviation. When in LCA we divide by the totality of the impact as normalization, up to a factor (the number of elements that make up the whole), we divide by the arithmetic mean. Implicitly, this says that we consider this arithmetic mean is a good descriptor of impacts, that we are in a "normal" world.

But the LCA practitioner is not working in a "normal" world. Uncertainty calculations are generally done with log-normal distribution laws. In an LCI database, the distribution of substance emissions commonly follows a log-normal law (Qin and Suh 2017). Some LCA results are interpreted in orders of magnitude (such as toxicities (Frischknecht and Jolliet 2019)) and therefore on a logarithmic scale. An LCA is the combination of elements following log-normal distributions. If no formal solution has been found for a sum of log-normal variables, there is a general agreement this is well approximated by a log-normal distribution (Beaulieu et al. 1995). Although the log-normal law is less intuitive than the normal law, this distribution frequently occurs in 
nature (abundance of species, concentrations of minerals in the earth's crust, concentrations of pollutants in the atmosphere, dose-response curves, etc.) (Limpert et al. 2001). We can reasonably assume the impacts of the set of products and services follow log-normal distributions.

\subsection{Geometric mean as the normalization reference}

This leads to thinking about normalization in a different way. The log-normal distribution is described by the geometric mean, not by the arithmetic one. The geometric mean is more robust to extreme values, but more importantly, it results from a multiplication, while the arithmetic mean is constructed from a sum. It therefore becomes possible to factorize and thus simplify the result.

As an illustration, let a reference system resulting from $n$ processes $(i \in\{1, \ldots, n\})$, with $m$ impacts $(j \in$ $\{1, \ldots, m\})$, let $f_{i}$ the final demand of the process $i$ and $h_{i, j}$ the impact $j$ of the process $i\left(h_{i, j}\right.$ results from the multiplication of all elementary flows of the process by the associated characterisation factors.). The arithmetic mean of each impact category for the reference system is

$$
M_{j}^{1}=\frac{1}{n} \sum_{i=1}^{n} f_{i} h_{i, j}
$$

and the geometric mean

$$
M_{j}^{0}=\left(\prod_{i=1}^{n} f_{i} h_{i, j}\right)^{\frac{1}{n}}=\left(\prod_{i=1}^{n} f_{i}\right)^{\frac{1}{n}} \times\left(\prod_{i=1}^{n} h_{i, j}\right)^{\frac{1}{n}}
$$

The term $\left(\prod_{i=1}^{n} f_{i}\right)^{\frac{1}{n}}$ is constant whatever the impact, therefore it can be removed without changing the proportions between the normalized impacts.

With $\left(\prod_{i=1}^{n} h_{i, j}\right)^{\frac{1}{n}}$, the geometric mean of $h_{i, j}$ instead of $f_{i} h_{i, j}$, we have a normalization reference value that we only calculate with the LCI database, without having to deal with the final demand for the reference. This approach ensures consistency between the modelling of the system under study and the normalization references. This approach is especially relevant for process-based LCA, but can also be used with EIO-LCA.

\section{Discussion}

Normalizing by the geometric mean of the inventory database raises several considerations that are worthy of discussion. We present below elements of responses to several remarks that can be made. 


\subsection{About the geometric mean}

First remark: Even if there are no data bias, normalizing with the geometric mean gives a different result than normalizing with the overall impact. Indeed, normalizing by geometric mean will not lead to the same conclusions as the "conventional" normalization, each normalization choice plays a key role in the aggregation/decision process as shown in MADM. However, it is worth noting that the results are identical with the assumption of a constant $\frac{M_{j}^{1}}{M_{j}^{0}}$ ratio regardless of $j$ (Hélias et al. 2020). They prove the results are highly correlated in some cases, despite data biases that can be explained. More precisely, assuming lognormal distribution, $\frac{M_{j}^{1}}{M_{j}^{0}}=\sqrt{1+C v_{j}}$ (see equation (10-11) in Limbrunner et al. (2000)) and the hypothesis of a constant ratio becomes a constant coefficient of variation $c v_{j}, \forall j$ (or at least small changes in the coefficient of variations).

Note in addition that the variability of the ratio $\frac{M_{j}^{1}}{M_{j}^{0}}$ is reduced by the square root with respect to $c v_{j}$. It would be interesting to investigate further the values of these ratios with the EIO-LCA databases where $M_{j}^{1}$ and $M_{j}^{0}$ are then computable from the same data for all impact categories.

Second remark: The geometric mean does not make the quantities commensurable. Indeed, for the purposes of normalization, the objective of being able to compare and communicate the results is not achieved by this normalization. This approach is interesting from the perspective of aggregating impacts. With some practice, reasoning with the geometric mean would be possible for an LCA practitioner but it would obviously be uncomfortable to communicate, the citizen-equivalent remaining the best solution for this purpose.

\subsection{About the inventory database as data for normalization}

Third remark: As the LCI database is used, this is not a true external normalization. In this approach, there is a link between the system studied and the references, but the building of both from the same source does not make it an internal normalization. The normalization here is not based on the case under study, but on the used database. It therefore goes beyond the internal normalization and can therefore be considered as an external normalization.

Fourth remark: No LCI database is perfect, some elements are missing and none represents the world as a whole. Over time, LCI databases are improved, but they are still perfectible. Using them to represent the world implies that the world is imperfectly modelled. But it is as imperfectly modelled as the system under study. Consider one modelled with a database where infrastructures are not represented, as the agrifootprint database (e.g. see Corrado et al. (2018)). Using a reference including infrastructure is not really relevant: the 
biases are potentially different according to the impacts (Heijungs et al. 2007), which will play a role in aggregation and may lead to biased conclusions. The best solution would obviously be a model of the system under study with infrastructures, but if missing they have to be missing in the reference as well. Consistency of the modelling is more important than the completeness of the reference for normalization purpose.

Fifth remark: The LCA practitioner uses several databases / the LCA practitioner modifies the database. It is always risky to make an LCA with heterogeneous databases that do not necessarily have the same perimeters. But in this case, it is enough to take as normalization value the geometrical mean of the union of the databases. If LCA practitioners substantially modify their databases, it is indeed necessary to recalculate the reference values and they will therefore have to do so (practitioners could also make their data public, these data would then be directly integrated into the inventory databases and the reference values would be calculated for them).

Sixth remark: The results will change if another LCI database is used. This is a necessity to ensure consistency between the system under study and the normalization reference. In this case, there is no longer a single set of normalization values linked to a life cycle impact assessment (LCIA) method, but rather a set for each database-LCIA method pair. An alternative would be to define a single reference resulting from the union of all the inventory databases, but this deserves further consideration.

\subsection{About planetary boundaries as normalization}

Seventh remark: With a normalization by planetary boundaries, the problem of consistency between inventory and reference seems solved. We have a real enthusiasm for the use of planetary boundaries in LCA (see Bjørn et al. (2020) for a review), Bjørn and Hauschild (2015) being probably the most emblematic corresponding work. The principle is to use the caring capacity as a normalization value instead of the global impact. Planetary boundaries are biophysical characteristics, not determined by human activities as it is the case for the system under study. Consequently, one might consider that it is not necessary to ascertain the consistency of the modelling. It is then possible to argue that it is not the planetary limit that is the value of interest, but whether or not it is exceeded, or close to it. In Bjørn and Hauschild (2015), this is addressed considering the normalization by planetary boundaries as a distance-to-target: Let $G_{j}$, the global impact $j, C_{j}$ the corresponding carrying capacity according to the planetary boundaries and $S_{j}$ the impact of the system under study. The normalization by the planetary boundary $\frac{S_{j}}{C_{j}}$ corresponds to the simplification of $\frac{S_{j}}{G_{j}} \times \frac{G_{j}}{C_{j}}$, with $\frac{S_{j}}{G_{j}}$ the result of the conventional normalization and $\frac{G_{j}}{C_{j}}$ the distance-to-target, named the reduction factor by Vargas-Gonzalez et al. 
1 (2019). This result makes it possible to be free of the conventional normalization reference $\left(G_{j}\right)$ and at first

2 glance, $S_{j}$ and $C_{j}$ do not therefore need to be modelled in the same way. However, two limitations can be raised.

3 The first one is a distance-to-target expressed in another way, with non-linearity (as squared with $\left(\frac{G_{j}}{C_{j}}\right)^{2}$ as it is

4 done in the ecoscarcity method (Frischknecht and Büsser Knöpfel 2013)), which makes this simplification

5 unfeasible. The second is that we only represent these elements by means of models. We try to determine $S_{j}$ but,

6 with our data, we only obtain an approximation, we will name $\hat{S}_{j}$. The same happens with $C_{j}$, our knowledge of

7 the involved mechanisms and our hypotheses only allow us to obtain a representation $\check{C}_{j}$. In $\frac{S_{j}}{G_{j}}$ the modelling of

8 the numerator must be consistent with the modelling of the denominator (i.e. $\frac{\hat{S}_{j}}{\hat{G}_{j}}$ ) and the same is true for $\frac{G_{j}}{C_{j}}$ with

$9 \quad \frac{\breve{G}_{j}}{\check{C}_{j}}$. As the system under study and the carrying capacity do not have the same processes of modelling, the

10 simplification $\left(\widehat{G}_{j}\right.$ versus $\left.\breve{G}_{j}\right)$ cannot be achieved. Unless to prove the similarities of the modelling, the problem

11 of consistency between the studied system and normalization remains with planetary boundaries. The

12 geometrical mean solves this problem.

\section{Conclusion}

Multi-criteria decision-making is a complex field of research and there is no universal method that can be applied in all cases. The LCA presents an interesting particularity, with normalization we generally use external information to express the attributes (i.e. impacts) into a common scale. This makes the multi-criteria decision process more generic, independent of the case study. The other side of the coin is the introduction of an additional bias via different modelling between the system under study and the reference, especially for processbased LCA. Normalization by the geometric mean of the inventory database avoids this bias. That is argued considering that log-normal laws govern the phenomena and processes under investigation. In addition to this normalization issue, describing the database by its geometric means and comparing them to the cumulative impact values is a way to see the exhaustiveness of the database (subject to the exhaustiveness of the normalization value, see Hélias et al. (2020)). This can be an additional step towards more robust and relevant LCAs, that best represent environmental impacts.

\section{Acknowledgements}

Authors thank C. Pradinaud for her valuable comments which helped improving this paper. 
Andreas R, Serenella S, Jungbluth N (2020) Normalization and weighting: the open challenge in LCA.

Int J Life Cycle Assess. https://doi.org/10.1007/s11367-020-01790-0

Beaulieu NC, Abu-Dayya AA, McLane PJ (1995) Estimating the distribution of a sum of independent lognormal random variables. IEEE Trans Commun 43:2869. https://doi.org/10.1109/26.477480

Bjørn A, Chandrakumar C, Boulay A-M, et al (2020) Review of life-cycle based methods for absolute environmental sustainability assessment and their applications. Environ Res Lett 15:083001. https://doi.org/10.1088/1748-9326/ab89d7

Bjørn A, Hauschild MZ (2015) Introducing carrying capacity-based normalisation in LCA: framework and development of references at midpoint level. Int J Life Cycle Assess 20:1005-1018. https://doi.org/10.1007/s11367-015-0899-2 Corrado S, Castellani V, Zampori L, Sala S (2018) Systematic analysis of secondary life cycle inventories when modelling agricultural production: A case study for arable crops. J Clean Prod 172:3990-4000. https://doi.org/10.1016/j.jclepro.2017.03.179

Fazio S, Castellani V, Sala S, et al (2018) Supporting information to the characterisation factors of recommended EF Life Cycle Impact Assessment method. EUR 28888 EN, European Commission, JRC109369, Ispra

Frischknecht R, Büsser Knöpfel S (2013) Swiss Eco-Factors 2013 according to the Ecological Scarcity Method. Methodological fundamentals and their application in Switzerland. Environmental studies no. 1330. Bern

Frischknecht R, Jolliet O (eds) (2019) Global Guidance for Life Cycle Impact Assessment Indicators: Volume 2. UNEP/SETAC Life Cycle Initiative

Gibbs EPJ (2014) The evolution of one health: A decade of progress and challenges for the future. Vet Rec 174:85-91. https://doi.org/10.1136/vr.g143

Heijungs R, Guinée J, Kleijn R, Rovers V (2007) Bias in normalization: Causes, consequences, detection and remedies. Int J Life Cycle Assess 12:211-216. https://doi.org/10.1065/lca2006.07.260

Hélias A, Esnouf A, Finkbeiner M (2020) Consistent normalization approach for Life Cycle Assessment based on inventory databases. Sci Total Environ 703:134583. https://doi.org/10.1016/j.scitotenv.2019.134583 
Jahan A, Edwards KL (2015) A state-of-the-art survey on the influence of normalization techniques in ranking: Improving the materials selection process in engineering design. Mater Des 65:335-342. https://doi.org/10.1016/j.matdes.2014.09.022

Laurent A, Hauschild MZ (2015) Normalisation. In: Hauschild MZ, Huijbregts1 MAJ (eds) Life cycle impact assessment. Springer Netherlands, Dordrecht, pp 271-300

Limbrunner JF, Vogel RM, Brown LC (2000) Estimation of Harmonic Mean of a Lognormal Variable. J Hydrol Eng 5:59-66. https://doi.org/10.1061/(ASCE)1084-0699(2000)5:1(59)

Limpert E, Stahel WA, Abbt M (2001) Log-normal Distributions across the Sciences: Keys and Clues. Bioscience 51:341-352. https://doi.org/10.1641/0006-3568(2001)051[0341:LNDATS]2.0.CO;2

Millennium Ecosystem Assessment (2005) Ecosystems and human well-being: Biodiversity synthesis. World Resources Institute, Washington D.C.

Muhl M, Berger M, Finkbeiner M (2021) Distance-to-target weighting in LCA-A matter of perspective. Int J Life Cycle Assess 26:114-126. https://doi.org/10.1007/s11367-020-01837-2 Myllyviita T, Leskinen P, Seppälä J (2014) Impact of normalisation, elicitation technique and background information on panel weighting results in life cycle assessment. Int J Life Cycle Assess 19:377-386. https://doi.org/10.1007/s11367-013-0645-6

Norris GA (2001) The requirement for congruence in normalization. Int J Life Cycle Assess 6:85. https://doi.org/10.1007/BF02977843

Pizzol M, Laurent A, Sala S, et al (2017) Normalisation and weighting in life cycle assessment: quo vadis? Int J Life Cycle Assess 22:853-866. https://doi.org/10.1007/s11367-016-1199-1

Prado V, Cinelli M, Ter Haar SF, et al (2019) Sensitivity to weighting in life cycle impact assessment (LCIA). Int J Life Cycle Assess. https://doi.org/10.1007/s11367-019-01718-3

Qin Y, Suh S (2017) What distribution function do life cycle inventories follow? Int J Life Cycle Assess 22:1138-1145. https://doi.org/10.1007/s11367-016-1224-4

UNEP (2020) United Nation, Department of Economic and Social Affairs, The 17 Goals. https://sdgs.un.org/goals. Accessed 29 Jul 2020

Vargas-Gonzalez M, Witte F, Martz P, et al (2019) Operational Life Cycle Impact Assessment weighting factors based on Planetary Boundaries: Applied to cosmetic products. Ecol Indic 107:105498. https://doi.org/10.1016/j.ecolind.2019.105498 
UNEP-SETAC Life Cycle Initiative. J Clean Prod 161:957-967. https://doi.org/10.1016/j.jclepro.2017.05.206

\section{Figure Caption}

7 
Figure

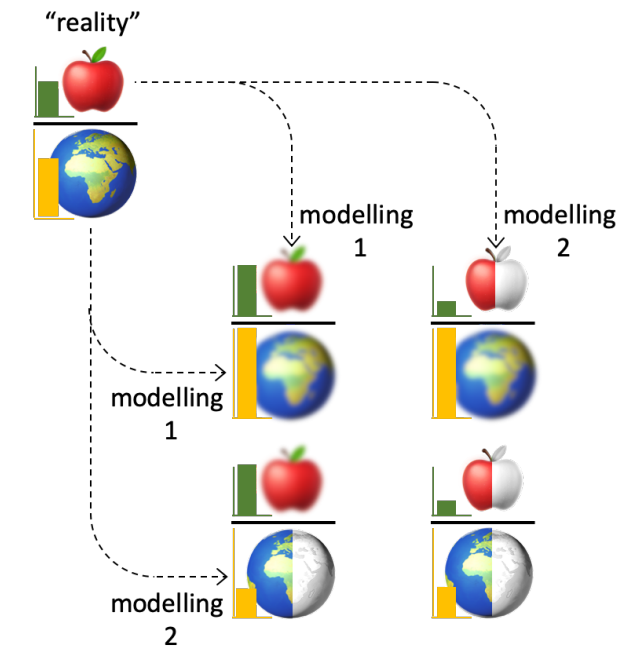

2

Figure 1 